\title{
Les Vies authentiques de peintres imaginaires de Beckford
}

L'imagination pittoresque à l'œuvre

\section{Luc Ruiz}

\section{OpenEdition \\ Journals}

Édition électronique

URL : http://journals.openedition.org/recherchestravaux/127

DOI : 10.4000/recherchestravaux.127

ISSN : 1969-6434

Éditeur

UGA Éditions/Université Grenoble Alpes

Édition imprimée

Date de publication : 15 avril 2006

Pagination : 15-27

ISBN : 2-9518254-8-X

ISSN : 0151-1874

Référence électronique

Luc Ruiz, "Les Vies authentiques de peintres imaginaires de Beckford», Recherches \& Travaux [En ligne], 68 | 2006, mis en ligne le 06 novembre 2008, consulté le 08 septembre 2020. URL : http://

journals.openedition.org/recherchestravaux/127 ; DOI : https://doi.org/10.4000/recherchestravaux. 127 


\section{Les Vies authentiques de peintres imaginaires de Beckford}

\section{L'imagination pittoresque à l'œuvre ${ }^{1}$}

La première chose qui frappe lorsqu'on commence à s'intéresser aux Vies authentiques de peintres imaginaires [VAPI] que Beckford publie en 1780, à vingt ans, c'est l'aura de mystère qui entoure le texte. L'œuvre constitue une sorte d'énigme. On en trouve la manifestation la plus nette dans la rareté des commentaires relatifs aux VAPI, y compris chez des critiques qui se sont attachés, pourtant de manière approfondie, à la vie de Beckford ou à ses productions. Pour l'illustrer, il suffit de prendre pour exemple quelques études qui appartiennent à la critique française: ni Didier Girard, dans une biographie assez récente (William Beckford. Terroriste au palais de la raison), ni Marc Chadourne (Eblis ou l'enfer de William Beckford), ni André Parreaux, dans un essai qui a fait date il y a plus de quarante ans (William Beckford, auteur de Vathek) ${ }^{2}$, n'accordent beaucoup de place à la première œuvre de celui que Byron qualifie d'«England Wealthiest son». C'est évidemment le

1. Toutes les citations du texte proviennent de William Beckford, Vies authentiques de peintres imaginaires, traduction, préface et notes de Roger Kann, José Corti, «Bibliothèque romantique", $\mathrm{n}^{\circ} 24,1990$. Nous renvoyons à ce texte par l'indication VAPI suivie du numéro de page. Les citations en anglais viennent de William Beckford, Biographical Memoirs of Extraordinary Painters (fac-similé de l'édition de 1780, introduction de Philip Ward), Londres, The Oleander Press, 1977, abréviation BMEP.

2. D. Girard, William Beckford. Terroriste au palais de la raison, José Corti, 1993; M. Chadourne, Eblis ou l'enfer de William Beckford, suivi d'une Anthologie de l'auvre en ses meilleures pages [dans laquelle ne figure aucun extrait des VAPI - ce qui en fait, du même coup, des pages «moins bonnes»], J.-J. Pauvert, 1967; A. Parreaux, William Beckford, auteur de Vathek (1760-1844). Étude de la création littéraire, Nizet, 1960. Il existe toutefois un remarquable article qui explore la complexité des VAPI: André Parreaux, "Les "peintres extraordinaires" de Beckford sont-ils une satire des écoles flamande et hollandaise?", Revue du Nord, n ${ }^{\circ} 169$, janvier-mars 1961. 
chef-d'œuvre, Vathek, conte arabe - qui plus est rédigé en français - qui éclipse le reste des écrits de celui qu'on a plaisamment (et significativement) surnommé de son vivant «le calife de Fonthill».

Les VAPI ont ainsi un statut particulier: elles apparaissent comme le premier fruit du talent d'un écrivain génial, mais dont l'œuvre majeure reste à venir (Vathek paraît en 1787); elles ne sont que le premier crayon d'un auteur de dix-sept ou dix-huit ans. De là à n'en faire qu'une pochade, il n'y a qu'un pas. Ainsi, selon le premier biographe de Beckford, Cyrus Redding, l'œuvre aurait été écrite dans l'intention de fournir un guide destiné à la domestique chargée de commenter les tableaux paternels aux visiteurs - plaisanterie qui est à double détente puisqu'elle a pour objet de multiplier les dindons de la farce ${ }^{3}$. Il se serait également agi de décalquer un des ouvrages de la bibliothèque paternelle, La Vie des peintres flamands de JeanBaptiste Descamps ${ }^{4}$, en surenchérissant sur le ridicule de certaines biographies de peintres ${ }^{5}$. S'il est évident que les VAPI ne sont pas dénuées d'intentions malicieuses, et même d'un certain sens de la facétie (elles sont souvent fort comiques), il est tout aussi indéniable qu'elles reprennent le modèle déjà constitué au XVIII siècle de la Vie de peintres; toutefois, on fait l'hypothèse que l'œuvre va très largement au-delà de ces données premières. On tentera de l'illustrer en montrant d'abord que les VAPI sont composites et reposent sur une esthétique du mélange, ensuite qu'en dépit de cette caractéristique, elles tissent des réseaux et constituent un ensemble cohérent; enfin qu'elles inventent un mode d'écriture en jouant, à leur manière, avec l'univers pictural qu'elles représentent.

Le statut composite des Vies authentiques: une esthétique du mélange

L' «Avertissement» qui précède les cinq biographies - semble-t-il de la main du précepteur de Beckford, le révérend John Lettice ${ }^{6}-$ n'installe pas $^{\prime}$ l'ouvrage dans une fiction préalable, comme le font certains romansmémoires du XVIII ${ }^{\mathrm{e}}$ siècle, ceux de Marivaux par exemple, dans lesquels un

3. C. Redding, Memoirs of William Beckford of Fonthill, Author of Vathek, 2 vol., Londres, Charles J. Skeet Publisher, 1859, vol. 1, p. 96-98.

4. J.-B. Descamps, Vie des peintres flamands, allemands et hollandois, Paris, 1753-1764. Voir la référence explicite de Beckford, en note, à la vie de Gérard Dou dans cet ouvrage [VAPI 119], Roger Kann montre ailleurs l'emprunt de l'auteur à Descamps [VAPI 99, note $1]$.

5. C. Redding, op. cit., p. 96.

6. Voir la «Préface» de Roger Kann [VAPI 12]. 
récit fictif vient justifier l'histoire à venir. Tout au contraire, l'éditeur joue habilement de l'ambiguïté, dans la continuité du titre anglais de l'ouvrage ${ }^{7}$, et, pour l'essentiel, insiste sur la jeunesse et les mérites d'un auteur (termes author et writer) qui garde l'anonymat. Mais cet auteur n'en reste pas moins potentiellement un véritable biographe (Biographical Memoirs dit le titre original).

La toute fin de l' "Avertissement", cependant, en arrive à définir le texte par rapport au roman. Il s'agit en effet pour l'écrivain, signale l'éditeur, de recourir à «un moyen plus original que celui habituellement suivi par les auteurs de romans.» [VAPI 16]. On notera au passage que le vocabulaire français ne permet de rendre qu'une partie de la phrase: «roman» recouvre en fait deux termes anglais, novel-writing et romance [BMEP s.p. (II)]. Quoi qu'il en soit, l'éditeur, in fine, reconnaît le caractère fictif de l'œuvre qu'il présente, mais il l'exclut dans le même mouvement du champ du romanesque, que celui-ci soit à tendance réaliste ou d'inspiration plus fabuleuse (Novel et Romance). Bref, la mention du roman apparaît sur le mode de l'exclusion. Dès lors, on peut légitimement se poser la question suivante: si le texte n'a rien de romanesque, quel est alors le «moyen plus original (a more original medium)» inventé par l'auteur? En un mot, quelle est la véritable nature de l'œuvre?

La question, on s'en doute, n'a pas de réponse simple. Le cadre général des VAPI est emprunté aux biographies de peintres sur le modèle de Descamps; mais cela suppose une double dimension perceptible dans le titre d'un ouvrage antérieur de Du Bois de Saint-Gelais, à la technique différente: Description des tableaux du Palais-Royal, avec la vie des peintres à la tête de leurs ouvrages $^{8}$. Il s'agit donc, chez Beckford comme pour Descamps dans une bien moindre mesure, d'intégrer le descriptif au narratif et non plus de les séparer comme chez Du Bois de Saint-Gelais qui privilégie la forme du catalogue. Mais là s'arrête la ressemblance dans la mesure où les VAPI ne s'en tiennent pas au factuel ou à l'examen de la technique des peintres; bref il y a un véritable travail d'écriture littéraire.

7. Le titre anglais, Biographical Memoirs of Extraordinary Painters joue sur la polysémie du terme "extraordinaire" (qui relève de l'exception/du prodige, du fabuleux), là où le titre français installe un paradoxe («authentiques»/« imaginaires»). En anglais, le titre est énigmatique, en français il dévoile d'emblée les cartes puisqu'il exhibe la fiction.

8. L.-F. Du Bois de Saint-Gelais, Description des tableaux du Palais-Royal, avec la vie des peintres à la tête de leurs ouvrages, d'Houry, 1727. Beckford connaît probablement cet ouvrage. 
Beckford utilise des matériaux d'une grande diversité: la biographie cède parfois la place au récit de voyage ( $\mathrm{Og}$ de Basan et ses compagnons faisant route vers la Sicile [VAPI 66-70]), à l'histoire de brigands (la vie du père de Blunderbussiana est évoquée avant celle du peintre, dans ce qui pourrait relever de la Rogue Literature [VAPI 83-85]), à la scène de drame bourgeois ou de tragédie domestique (les reproches de sa femme au père d'Aldrovandus qui veut l'envoyer commercer avec les pays du Levant [VAPI 18-21]). L'auteur décrit des situations qui semblent tout droit sorties d'histoires comiques du $\mathrm{XVII}^{\mathrm{e}}$ siècle (le goût qu'éprouve Mierhop pour les poissons et les pièces de viande, aussi bien pour les peindre que pour les manger [VAPI 97]), de la plume du satiriste (la description de la politesse ridicule du cercle de Monsieur Baise-la-main [VAPI 105-107]) ou du conte de fées (l'édit interdisant de conserver de la toile rappelle la prohibition des fuseaux dans La Belle au bois dormant de Perrault - et il est tout aussi inefficace [VAPI 31]). Cette variété s'accompagne d'une multitude de notations ou de situations incongrues (le squelette de Blunderbussiana est canonisé par la faculté! [VAPI 92]) qui ne manquent pas de déclencher l'hilarité: l'humour fait très fréquemment dériver le texte vers l'extravagance.

En termes génériques (ou sub-génériques), les VAPI constituent un assemblage très complexe; on sent à la lecture que l'œuvre est pétrie de références, qu'elle est dans un flottement perpétuel entre diverses tentations ou différents possibles.

Une telle description des VAPI peut donner l'image d'une œuvre discontinue, hétéroclite, d'un collage d'éléments disparates, bref d'une «insipide rhapsodie» - pour reprendre les termes utilisés par le lecteur fictif devant la narration de Jacques le fataliste ${ }^{9}$. Il semble bien que Beckford, d'ailleurs, ait facétieusement inscrit cette possibilité dans le patronyme d'un de ses peintres. Le nom de Blunderbussiana, héros de la quatrième vie, semble bien associer "lourdaud, maladroit» et "ana", l'affixe qui signale les collections de pensées détachées, de bons mots, d'anecdotes diverses - sur le modèle de "Virgiliana ". Le terme serait alors l'équivalent de Maladroitiana, à entendre comme un recueil de pensées sur une série de lourdauds, pire comme un recueil compilé par un auteur balourd...

Or, il n'en est rien, les VAPI sont à mille lieues d'un texte maladroit; elles semblent au contraire une entreprise très concertée dans la mesure où elles font de ces cinq récits composites et indépendants un univers cohérent.

9. Diderot, Jacques le fataliste, Librairie générale française, «Le Livre de Poche classique», 2000, p. 285. 


\section{Un tissage de réseaux : relations et entrecroisements}

À première vue, les cinq vies de peintres sont indépendantes: des parcours bien distincts sont présentés au lecteur. Les cinq sections, dotées d'un titre spécifique, en témoignent. De même, chacun des cinq peintres est ancré dans un lieu d'origine: successivement Bruges, Basan (en Poméranie), Vienne, la Dalmatie, Amsterdam. Chaque parcours, enfin, est assez nettement individualisé par le talent singulier de chaque peintre. Le récit le plus long, toutefois, déroge à cette règle générale: il s'agit de la seconde biographie qui est double, puisqu'elle rapporte l'histoire de deux parents, André Guelph et $\mathrm{Og}$ de Basan dont les récits de vie sont entrelacés. On peut se demander si la structure de cette deuxième vie, unique dans le recueil, n'a pas en quelque sorte valeur d'indice.

En effet, on s'aperçoit aisément que les récits sont loin d'être imperméables les uns aux autres; au contraire, toute une série d'éléments constitue autant de ponts entre eux. C'est ce qu'indique le biographe lorsqu'il affirme que la parenté d'André et $\mathrm{Og}$ n'est qu'une relation superficielle, que ce qui les lie est beaucoup plus fort (en l'occurrence, il s'agit du désir de représenter) ${ }^{10}$. Mais l'œuvre met en avant un autre lien entre les protagonistes, il s'agit de la relation entre maître et élève. Prenons un seul exemple parmi bien d'autres ${ }^{11}$ : le premier récit nous apprend qu'Aldrovandus Magnus refuse tout enseignement, sauf à André Guelph et $\mathrm{Og}$ de Basan qui prolongeront son ouvre [VAPI 30]. Autre exemple de lien: la confrontation entre peintres. Héritiers de la technique d'Aldrovandus, Og et André vont s'opposer à deux de leurs pairs, Sucrewasser de Vienne et Soorcrout, qui préconisent des moyens artistiques différents: Beckford en tire l'épisode savoureux de la controverse sur l'utilisation des vernis à l'huile de noix et au blanc d'œuf; à Venise, une assemblée de spécialistes reconnaîtra la supériorité de l'huile de noix [VAPI 42-46]. La cohérence d'ensemble des trois premières vies est ainsi assurée par la rencontre des personnages (les peintres apparaissent dans un récit antérieur avant que leur vie soit racontée dans le récit sui-

10. «L'étroite parenté entre Og et André leur donnait maintes occasions de se rencontrer mais bien plus que les liens du sang, un même naturel les unissait et un même état les amenait souvent à se rendre aux champs. Le plaisir mutuel qu'ils prenaient à contempler la nature leur fit très tôt désirer d'en représenter les beautés " [VAPI 36].

11. La relation entre maître et élève apparaît dans chacune des vies. Aldrovandus Magnus a pour maître Hemmeline; Og de Basan aura à son tour un élève, Benboaro Benbacaio (qui a abandonné l'enseignement de Jules Romain pour cause de divergence); Sucrewasser est le disciple d'Insignificanti (avant de se brouiller avec lui), Blunderbussiana de Joseph Porta, enfin Watersouchy de Mierhop, puis de Gérard Dou. 
vant) et par la nature de leurs relations: affinité entre Aldrovandus et ses élèves André et $\mathrm{Og}$; rivalité entre ces derniers et le couple formé par Sucrewasser et Soorcrout.

Ainsi, les deux derniers récits se situent en marge: les quatrième et cinquième biographies ne croisent aucune autre, elles sont par conséquent autonomes. Blunderblussiana, l'ancien bandit des montagnes dalmates, bien qu'il s'installe à Venise, ne fréquente pas les autres peintres; Watersouchy, quant à lui, passe toute sa carrière à Amsterdam, Leyde et Anvers. Cependant Beckford prend soin de les relier aux autres artistes d'une manière subtile. La vie de Sucrewasser commence par un discours du biographe sur l'organisation du recueil et la personnalité des peintres qui vaut la peine d'être cité:

Nous allons à présent évoquer des faits et des circonstances largement différents de ceux que nous venons de décrire. Nous ne représenterons plus des artistes consumés par la flamme du génie ou égarés par l'ardeur de l'imagination. Nos lecteurs, tout au moins les plus sages et les plus avisés, vont pouvoir admirer maintenant la conduite rangée et pleine de bon sens d'un Sucrewasser, en opposition flagrante avec les extravagances d'un Og. [VAPI 77]

Deux catégories semblent être mises en place ici: les excentriques et les raisonnables. Il est suggéré que le recueil repose sur ces deux types de caractères, qu'il est bipartite. D'un côté les génies en proie à leur imagination (Aldrovandus Magnus, André Guelph et Og de Basan), de l'autre des peintres à l'attitude moins extrémiste, plus régulière (Sucrewasser de Vienne et son ami pour un temps Soorcrout, Blunderbussiana, et enfin Watersouchy).

Or, le lecteur avisé qui fait bien son travail (c'est le biographe qui lui suggère de le faire) constate, pour plusieurs raisons, que cette division est erronée, qu'elle ne correspond pas au texte. Par exemple, un peintre comme Blunderbussiana, par ses excès, mais sans doute aussi par la thématique de ses œuvres (des paysages sauvages, des scènes saisissantes, [VAPI 90-91]), est beaucoup plus proche du premier groupe de peintres, notamment de Og. De son côté, André Guelph apparaît en bien des occasions raisonnable: il tempère à plusieurs reprises les ardeurs du fougueux Og. Enfin, l'extravagance est bien loin d'être étrangère aux artistes du second ensemble, il semble seulement qu'elle se trouve ailleurs: par exemple, dans un soin particulier attaché au détail qui fait de chacun d'eux un génie en son genre. Ainsi, Sucrewasser se distingue comme peintre de blasons, il y excelle dans les pattes de lions, les queues de dragons et les plumages d'aigles [VAPI 78]; Blunderbussiana produit des œuvres "d'un dessin miraculeux» en représentant les tendons et les artères des cadavres disséqués de ses propres victimes [VAPI 87-88]; Watersouchy s'épuise à mettre «les dernières touches aux 
doigts de sa protectrice» dont il fait le portrait: cette opération, pour porter à la perfection la représentation des doigts, dure à elle seule un mois... [VAPI 119].

Les remarques du biographe font donc ici office de trompe-l'œil puisqu'elles sont partielles, sinon erronées. L'attention n'est pas moins attirée sur les rapports et sur les contrastes entre les peintres. On peut se demander si à travers la structure du recueil indiquée par le biographe, Beckford ne propose pas une sorte de jeu au lecteur: la recherche d'éléments susceptibles d'établir des rapprochements.

Parmi ces indices, les jeux onomastiques occupent une place de choix. Certains noms permettent de constituer une série, celle de la nourriture: tout le monde comprend que Sucrewasser peut se traduire par "eau sucrée», Soorcrout par "choucroute», quant à Watersouchy, signale Roger Kann, il renvoie au "waterzootje» néerlandais, une sorte de poule en ragoût; mais le "water souchy» est également un plat de poisson cuit et servi dans un peu d'eau ${ }^{12}$ - ce qui est un comble pour un peintre né sur les canaux d'Amsterdam ${ }^{13}$. Ces noms ridicules correspondent aux peintres obsédés par le détail et très exactement à ceux que le biographe se permet de critiquer ouvertement, ce qui est assez rare dans l'œuvre, pour leur «bassesse» dans le cas de Sucrewasser et Soorcrout [VAPI 42], pour sa "prétention insupportable» dans celui de Watersouchy [VAPI 117]. Les ranger du côté de la nourriture revient à les placer du côté de la matérialité, de la matière première - ce qui n'est pas sans rapport avec leur peinture, comme dans le cas du portrait à l'exactitude extrême du bourgmestre Van Gulph réalisé par Watersouchy (ibid.).

À l'opposé, les patronymes des autres peintres jouent sur des connotations différentes: Aldrovandus ${ }^{14}$ se voit accorder le titre de Magnus et est comparé, dans une épitaphe, à Alexandre le Grand (au demeurant, le passage est fort comique) [VAPI 31-33]; le nom d'André Guelph rappelle l'opposi-

12. "A dish consisting of small fish stewed and served in a little water. /Written also water sou chet].", Webster Dictionary, édition 1913, p. 1633.

13. Dans Voyage d'un rêveur éveillé de Londres à Venise (t. 1), les lettres qu'il rédige durant son Grand Tour sur le continent, Beckford développe une théorie très amusante sur «le côté ichtyologique des Hollandais", José Corti, «Bibliothèque romantique», $\mathrm{n}^{\circ}$ 17, 1988, p. 65.

14. Un médecin, naturaliste et botaniste du XVI siècle porte ce nom: Ulisse Aldrovandi (ou Aldrovandus, 1522-1605). Il serait l'auteur, entre autres, d'une Histoire naturelle des ser pents et des dragons. Or Aldrovandus (qui se prénomme Antoine) est l'auteur d'un tableau qui raconte "la pieuse histoire de La Belle et du Dragon" [VAPI 24], en anglais "the sacred story of Bell and the Dragon" [BMEP 11]. C'est là, sans doute, une erreur de traduction. Beckford s'amuse à introduire une référence biblique. Il fait ici une allusion à un épisode de l'Ancien Testament, l'histoire de Bel et du dragon, parfois appelée «Bel et le serpent» (Daniel, 14). 
tion des guelfes et des gibelins dans l'Italie des XIII et XIV ${ }^{e}$ siècles ${ }^{15}$; quant à Og de Basan, les sonorités de son nom suffisent à lui conférer une certaine noblesse. Mais Og de Basan (ou de Bashân) est en outre un roi biblique, exterminé avec son peuple par Moïse sur l'ordre de Dieu ${ }^{16}$. Ces noms, on le voit, ne manquent pas d'allure; en renvoyant à l'histoire, aussi bien réelle que biblique, ils assurent d'emblée ces personnages d'une destinée grandiose et presque tragique.

Cette double série de patronymes, qui oppose plaisamment matière alimentaire à matière historique, transpose à l'échelle des noms la distinction entre peinture d'histoire et peinture de genre ${ }^{17}$. Bien sûr, cette division ne recoupe pas exactement les tableaux réalisés par les peintres: André, spécialiste de botanique, peint des plantes et des paysages [VAPI 39], genre qu'Og pratique aussi [VAPI 41]; Sucrewasser, de son côté, réalise son chef-d'œuvre avec Salomé mère des Macchabées [VAPI 82]. Il n'en reste pas moins que Beckford semble vouloir jouer à établir, dans la sphère littéraire, des équivalences avec l'univers pictural.

L'ambition est non seulement de présenter un monde cohérent, régi par des règles de composition (ressemblances et oppositions), mais encore de brosser un tableau complet de l'univers pictural avec des moyens littéraires.

\section{L'invention d'un mode d'écriture: un récit qui joue avec le style pictural}

Au total, on peut se demander si les VAPI ne sont pas, en quelque sorte, un cabinet de peinture comme ceux que Beckford décrit parfois dans Voyage

15. L'analogie est d'autant plus frappante que l'orthographe anglaise de "guelphe» coïncide avec le nom du personnage.

16. Deutéronome 3, 3-4: "Yahvé notre Dieu livra aussi en notre pouvoir Og, roi du Bashân, et tout son peuple. Nous le battîmes si bien que pas un n'en réchappa». Le premier discours de Moïse résume le parcours des tribus d'Israël depuis le départ du Sinaï. Le royaume de Bashân (avec l'Arnon et Heshbon) fait partie des divers territoires de la rive orientale du Jourdain qui ont été conquis par Moïse. Il est donc inscrit dans le nom même du peintre d'être voué à la destruction par une sorte de volonté divine. C'est d'autant plus vrai que le Og biblique est le dernier des Réphaïm (Deutéronome 3, 11 ; il s'agit de géants), peuple que Yahvé a exterminé.

17. Voir la définition de Diderot dans l'Essai sur la peinture (1765): «On appelle du nom de peintres de genre, indistinctement, et ceux qui ne s'occupent que des fleurs, des fruits, des animaux, des bois, des forêts, des montagnes, et ceux qui empruntent leurs scènes de la vie commune et domestique [...] ", dans Euvres, Gallimard, "Bibliothèque de la Pléiade», 1951, p. 1159. Diderot conteste d'ailleurs dans la même page la distinction entre peintres d'histoire et peintres de genre et tente de la redéfinir. 
d'un rêveur éveillé, son œuvre suivante. Pour que la métaphore soit pertinente, il faudrait peut-être dire, une galerie, non de peintures, mais de peintres. La galerie est agencée dans des séries, on l'a vu précédemment. Elle s'organise également dans un ordre chronologique. Dans ce recueil de vies, où, à dessein, les dates sont rarement précisées ${ }^{18}$, quelques indices nous montrent que les récits se succèdent à peu près chronologiquement: Aldrovandus naît en 1473, Og est baptisé en 1519, les parents de Watersouchy se marient en $1640 \ldots$ La période couverte est donc d'environ deux cents ans.

Le lecteur objectera sans doute qu'il est un peu facile d'utiliser le vocabulaire pictural pour qualifier le texte des $V A P I$, à quoi on pourra répondre que l' "Avertissement» lui-même ne se prive pas de le faire: l'éditeur y annonce que "l'écrivain a d'ailleurs eu pour dessein (design ${ }^{19}$ ) de montrer des exemples caractéristiques de la nature et de l'art tout en les accompagnant de quelques esquisses (sketches) de nos mœurs" [VAPI 16]. Si l'éditeur fait de l'auteur un peintre par le vocabulaire employé, le biographe, dont les interventions font presque un personnage à part entière, ne manque pas de mettre en relation son écriture et la peinture en certaines occasions. On en a un exemple, avec une formule comme: "Que mes lecteurs se représentent (figure to themselves) Monsieur Baise-la-main menant l'obséquieux Watersouchy à travers de vastes salles et de longs couloirs [...] " [VAPI 106-107]. L'imagination picturale du lecteur est sollicitée, il est amené à se figurer la scène par des images. Mais cet appel montre peut-être également les limites du texte qui ne peut vraiment donner à voir. Le biographe le signale en plusieurs occasions. À propos d'un tableau réalisé par Watersouchy, la "maison de banque» de Monsieur Baise-la-main, il indique: "Il serait vain de donner par des mots une juste idée de ce chef-d'œuvre. Il faudrait l'avoir vu pour pouvoir l'admirer.» [VAPI 115]. Ailleurs, il déplore le manque d'espace pour décrire un tableau magistral:

[...] voilà qui demanderait au moins une cinquantaine de pages. Dans un aussi vaste espace on pou[rr]ait sans doute fixer autant de détails mais les mots manquent pour donner une idée juste de l'atmosphère de fête qui régnait sur l'ensemble (surpasses the power of words).» [VAPI 101]

18. Il faut parfois procéder par recoupements pour situer des dates ou des plages temporelles, par exemple en se fiant aux dates de naissance et de mort des véritables peintres évoqués dans le texte.

19. L'étymologie du terme, "desseigner» (de l'italien disegnare) renvoie à l'idée de dessiner. 
La peinture serait-elle alors impossible à rendre par les mots? Il semble bien que ce soit le cas. Toutefois, on mesurera l'humour sous-jacent des VAPI: ces toiles sont d'autant plus indescriptibles qu'elles n'existent pas. Plus sérieusement, il ne s'agit pas, pour l'écrivain, de rivaliser avec la peinture, mais de dresser une composition spécifique qui embrasse l'œuvre picturale et son exécutant. Une galerie de peintres, c'est-à-dire de personnages et pas seulement de toiles, constitue un magnifique support pour la satire: aussi sublimes que soient les œuvres des peintres, ils n'en sont pas moins hommes et susceptibles d'être satirisés pour leurs manies [VAPI 99-100], leur goût de l'argent ou leur vanité [VAPI 117]. Beckford joue ainsi à merveille sur les deux tableaux du pictural et du biographique.

Les VAPI traitent en fait chacun des récits comme une entité qui réunit dans un même tableau la biographie du peintre et son œuvre; mais c'est la vie des peintres elle-même qui est élevée au rang d'œuvre d'art. C'est visible, par exemple, dans l'unité profonde des récits qui non seulement constituent en destinée les biographies, mais cherchent également à épouser, jusque dans le ton et le style employés, la nature des événements vécus. Ainsi, Sucrewasser se définit en grande partie en conformité avec son nom: il est fils d'épiciers, se caractérise par sa douceur (" [il] ne désobéissait jamais» [VAPI 79]), sa peinture elle-même est douceâtre, y compris lorsqu'il doit représenter les Furies (« les serpents enroulés autour de leurs têtes avaient l'aspect inoffensif des anguilles» [VAPI 81]), il est qualifié de "pittore amabile» et sa mort, résultat "d'un refroidissement contracté lors d'une fête sur l'eau» à Venise, illustre bien que le sucre se dissout dans le milieu aqueux [VAPI 82]. Radicalement à l'opposé, Blunderbussiana, dont le nom renvoie à "blunder buss» à la fois «maladroit, lourdaud», on l'a dit, et «tromblon, espingole», mène une vie irrégulière et sauvage dans un milieu hostile; peintre autodidacte, il se spécialise dans la représentation de corps disséqués et de scènes violentes et torturées [VAPI 90-91]; cela le conduit à une agonie fiévreuse et frénétique durant laquelle il se croit au milieu de la danse macabre de tous les «membres mutilés qu'il [a] disséqués» [VAPI 92]; le biographe rapporte évidemment les événements avec le plus parfait humour noir.

Presque immanquablement, dans le recueil, la mort des artistes vient couronner leur vie, mais également leur œuvre qu'elle parachève en quelque sorte: Aldrovandus, privé de toile par un incendie, ne peut peindre comme il l'a prévu les exploits du prince Drahomir (englouti par un tremblement de terre) et expire un peu à la manière de ce héros, en prononçant les ultima

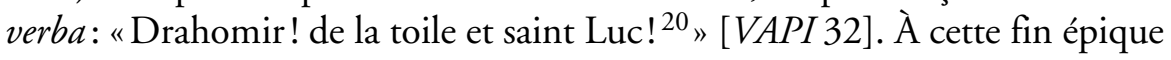

20. On convient habituellement que saint Luc était médecin, peut-être est-ce une manière 
s'opposent, à l'autre bout des VAPI, les dernières productions de Watersouchy, un fromage avec ses acariens (des cirons, et non des "vers" comme le dit la traduction française) et «le portrait d'une puce» [VAPI 120]. À ce resserrement extrême du sujet pictural correspond la mort du peintre qui s'étiole jusqu'à, littéralement, disparaître: "Il maigrit jusqu'à paraître transparent (he almost shrunk away to nothing)» (ibid.). On notera que ces deux vies de peintres donnent la structure globale de l'ouvrage: s'ouvrant en fanfare sur la démesure, la vigueur du trait, le grand (qu'on pense à Aldrovandus Magnus), il se clôt sur le minuscule, l'extinction et l'effacement.

Au total, il y aurait là deux formes de pittoresque: l'une renvoyant à l'idée de peinture bien caractérisée, frappante (sens 2 du Littré), l'autre insistant davantage sur les oppositions et les contrastes (sens 3 du Littré $^{21}$ ) - on rejoint en ce point certaines remarques de la partie précédente.

Mais Beckford va sans doute plus loin. Au cœur de l'œuvre se trouve la question de la représentation. En quelques occasions, les VAPI signalent le pouvoir d'illusion de la peinture, qui peut tromper le spectateur jusqu'à lui faire prendre pour réelle une simple image. Le biographe le laisse entendre lorsque, après avoir indiqué le sujet des deux premières toiles de Watersouchy, un fauteuil de velours et un tapis de Turquie, il ajoute: "On rêvait de s'asseoir dans le premier et les chiens de se rouler sur le second ${ }^{22}$ " [VAPI 102]. Ailleurs, c'est une anecdote qui traduit la même idée: devant un Moïse et le buisson ardent qu'Aldrovandus est en train de peindre, une jeune princesse s'écrie: «Oh! Maman, je ne veux pas toucher au buisson de peur de me brûler les doigts" [VAPI 26]. Sous la remarque du biographe ou cette scène, qui sont des variations humoristiques sur l'histoire des raisins de

de demander le secours d'un homme de l'art. Mais il est beaucoup plus probable qu'Aldrovandus invoque saint Luc en qualité de patron des peintres.

21. Littré (1872), article "pittoresque»: "1) Qui concerne la peinture. La composition pittoresque a ses règles. 2) Il se dit de tout ce qui se prête à faire une peinture bien caractérisée, et qui frappe et charme tout à la fois les yeux et l'esprit. Virgile est plein de ces expressions pittoresques dont il enrichit la belle langue latine, VolT. Dict. phil. Imagination. Les effets pittoresques et le bon goût du dessin, J.-J. Rouss. Ém. [...]. 3) Plus particulièrement se dit, depuis un demi-siècle, de ce qui résulte, en peinture, de l'opposition des lignes et du contraste brusque de la lumière et des ombres. / Il se dit dans un sens analogue des œuvres littéraires. Style pittoresque $[\ldots] »$.

22. Même jeu dans Voyage d'un rêveur éveillé où, évoquant la visite d'un cabinet, Beckford dit: "[...] son principal ornement était un chardon des plus sublimes peint par Snyders, d'une taille si héroïque, d'une imitation si fidèle qu'il mettrait un âne au comble de l'émotion», op. cit., p. 50. Ce peintre (1579-1657) est «la figure majeure de la peinture d'animaux et de natures mortes de chasse si florissante en Flandre au XVII e siècle», Encyclopaedia univer salis, art. «Frans Snyders». 
Zeuxis chez Pline l'Ancien, se dessine la question sérieuse des frontières du réel et de la représentation.

L'illusion que produit la peinture, Beckford la reproduit dans ses récits, à sa manière. Bien sûr, les vies de ses peintres sont imaginaires, mais des êtres de fiction y croisent des personnes qui ont existé: Jean Hemmeline ${ }^{23}$ voisine avec Joseph Porta, Benboaro Benbacaio côtoie Jules Romain, Insignificanti est à trois pages du Titien. La référence picturale, déjà riche (ce sont non seulement des peintres, mais également des sujets de toiles, ou encore des techniques) se complique de références historiques et littéraires (par exemple à Virgile [VAPI 69] ou à l'Arioste [VAPI 48-51]). Bref, les modèles, et par conséquent les lectures, sont multiples. Un exemple suffira, espérons-le, à le montrer: Og de Basan, sans doute un des plus complexes personnages de peintre dans l'œuvre, emprunte son nom à un roi qui apparaît fugitivement dans l'Ancien Testament. Mais «Bassan », cette fois avec deux «s», est la traduction française de Bassano qui renvoie à une famille de peintres originaire de cette ville italienne, les Da Ponte. Francesco Bassano ( 1549 1592) est, comme Og, l'auteur d'une ouvre ayant pour sujet L'Arche de Noé (palais ducal, Venise). Par ailleurs, le suicide d'Og, qui se jette dans l'Etna, rappelle la mort d'Empédocle, ce qui confere à ce peintre, également auteur de divers écrits, dont une dissertation sur la pluralité des mondes [VAPI 52], une aura philosophique... Mais comment meurt donc le bien réel Francesco Bassano? Louis-François du Bois de Saint-Gelais nous le rapporte: le cerveau attaqué par la mélancolie, sujet à des craintes continuelles, reclus dans sa maison, un jour, «il se jeta par la fenêtre, \& s'étant cassé la tête, il mourut peu de temps après ${ }^{24}$ ».

Beckford se plaît à multiplier les références et, surtout, à les brouiller avec une telle virtuosité qu'on ne sait plus où arrêter le jeu, qu'on ne sait même pas si l'on est parvenu au bout de l'interprétation.

À l'issue de ce parcours, on voit bien pourquoi la fiction biographique de Beckford est si complexe: elle croise l'univers pictural et, tout en en exhibant les codes, elle s'amuse à les transposer. Élever la vie imaginaire au rang d'œuvre d'art, voilà bien le projet. Mais un des mérites de ce petit livre réside dans sa manière: à la fois parodie, satire et conte extravagant, il est en perma-

23. Le nom, ici, est simplement altéré, Descamps rapporte la vie d'un Hans (Jean) Hemmelinck. Certains détails confirment qu'il est bien le Hemmeline de Beckford, par exemple sa naissance à Damme, près de Bruges.

24. L.-F. du Bois de Saint-Gelais, op. cit., p. 138. 
nence animé par un humour roboratif. Si Beckford pose des questions sérieuses, il le fait toujours avec légèreté; s'il avance des réponses, elles sont évasives.

Le lecteur, finalement, se retrouve devant le texte des VAPI comme le biographe devant la façade de l'hôtel particulier de Monsieur Baise-la-main. Il peut se dire: «je demeure confronté à "ses nombreuses figures allégoriques dont je ne suis jamais parvenu à comprendre l'exacte signification” " [VAPI 106]. Toutefois, cela ne l'empêche en rien de goûter le spectacle.

On comprend pourquoi Borges admirait Beckford ${ }^{25}$ : tous deux sont amateurs de labyrinthes et autres impossibles architectures ${ }^{26}$.

25. Voir "Sur le Vathek de William Beckford" dans Autres inquisitions, J.-L. Borges, Euvres complètes, t. 1, Gallimard, "Bibliothèque de la Pléiade», 1993, p. 771-774. Ce texte bref débute par une réflexion magistrale - et vertigineuse - sur la nature de toute entreprise biographique.

26. Nous nous permettons de renvoyer à un de nos articles qui reprend et développe certaines perspectives de cette contribution: «Comment rendre le biographique extraordinaire? Sur les Vies authentiques de peintres imaginaires de Beckford", Otrante, $\mathrm{n}^{\circ} 17$, "Théâtre \& fantastique. Une autre scène du vivant», printemps 2005, p. 205-217. 